\title{
Tilmicosina nas rações de suínos em fases de crescimento e terminação
}

\author{
Tilmicosine in rations of swines in growing and finishing phases
}

\author{
Caio Abércio da Silva ${ }^{1 *}$; Edgard Hideaki Hoshi² ${ }^{2}$ Juliana Sarubbi $^{3}$
}

\section{Resumo}

\begin{abstract}
O experimento foi conduzido visando avaliar o uso do antibiótico Tilmicosina nas concentrações de 100 ppm ou de 200ppm e o uso de Tiamulina (50 ppm) mais Cloridrato de Oxitetraciclina (150 ppm) no controle de doenças respiratórias principalmente causadas pelo Mycoplasma hyopneumoniae em suínos nas fases de crescimento e terminação. Foram utilizadas duas granjas comerciais, denominadas A e B, com distintos padrões sanitários. Utilizou-se no 945 animais, sendo 639 da granja A e 306 da granja B. Avaliou-se o desempenho dos animais nas fases de crescimento e terminação, a classificação (escore) de lesões nos pulmões, o Índice de Eficiência Econômica e o Índice de Custos Médios dos tratamentos experimentais. Não houve diferença $(\mathrm{P}>0,05)$ entre os tratamentos quanto ao desempenho zootécnico e ao escore de lesões nos pulmões. A concentração de $100 \mathrm{ppm}$ de Tilmicosina foi eficiente e economicamente viável no controle da pneumonia micoplasmática.

Palavras-chave: Antibióticos, desempenho, oxitetraciclina, pneumonia, suínos, tiamulina.
\end{abstract}

\begin{abstract}
The experiment was conducted to evaluate the use of $100 \mathrm{ppm}$ of Tilmicosine antibiotic or $200 \mathrm{ppm}$ and the use of Tiamulin $(50 \mathrm{ppm})$ plus Oxitetracicline Cloridate $(150 \mathrm{ppm})$ to control of respiratories diseases caused mainly by Mycoplasma hyopneumoniae in swines in growing and finishing phases. The experiment used two pigs farms (A and B) with different sanitaries standarts. It was used 945 swines, been 639 animals in farm A and 306 animals in farm B. The experiment evaluated the performance of animals in the growing and finishing phases, the classification (score) of lesions in lungs, and the economic efficiency and the costs of the experimental treatments. There were no difference $(\mathrm{P}>0.05)$ between the treatments to the performance and to the lesions scores in lungs for A and B farms. The concentration of $100 \mathrm{ppm}$ of Tilmicosine was efficient and economically viable to the control of mycoplasmal pneumonia
\end{abstract}

Key words: Antibiotics, oxitetracicline, performance, pneumonia, swine, tiamulin.

\section{Introdução}

O confinamento de suínos, tradicionalmente utilizado nas modernas granjas, exerce uma grande pressão sobre o animal, aumentando o estresse e favorecendo a instalação de agentes patogênicos como o Mycoplasma hyopneumoniae, causador da Pneumonia Enzoótica dos suínos. Esta patologia acomete clinicamente, principalmente, animais em crescimento e terminação e acarreta elevados prejuízos econômicos, piorando o índice de conversão alimentar e os gastos adicionais com ração (SILVA

\footnotetext{
1 Professor do Departamento de Zootecnia, Centro de Ciências Agrárias, Universidade Estadual de Londrina, Caixa Postal 6001, 86051-970, Londrina - PR. e-mail: casilva@uel.br.

2 Médico Veterinário, mestrando em Produção Animal da Universidade Estadual de Londrina.

3 Aluna de Graduação de Medicina Veterinária da Universidade Estadual de Londrina.

* Autor para correspondência.
} 
et al., 2001). Perdas econômicas com a Pneumonia Enzoótica podem chegar a 20\% sobre a conversão alimentar e até 30\% sobre o ganho de peso (SOBESTIANSKY et al., 1999). A taxa de morbidade tem variado de 40 a $60 \%$ para animais entre 4 a 6 meses de idade e a mortalidade pode chegar a 5\% quando a Pneumonia Enzoótica está instalada no sistema de produção de suínos .

A utilização do sistema contínuo, a alta densidade de animais por baia e o deficiente manejo sanitário têm tido grande influência na predisposição às patologias respiratórias (PIFFER, 1983).

Normalmente, a infecção inicia-se com a aquisição de animais positivos e esta se mantém na granja através dos portadores assintomáticos (PIFFER, 1998).

A pneumonia micoplasmática é a forma primária que, diminuindo as defesas respiratórias, favorece a ação de agentes secundários como a Pasteurella multocida, o Haemophilus parasuis e o Actinobacillus pleuropneumoniae (STEVENSON, 1998).

O Mycoplasma hyopneumoniae é um agente de difícil eliminação, sendo importante, portanto, conviver com a doença sob níveis economicamente satisfatórios, mantendo a aplicação de medidas terapêuticas e imunoprofiláticas e a correção constante dos fatores de risco (RISTOW et al., 2001).

Programas sanitários que utilizam antimicrobianos continuamente nas rações ou em épocas específicas, sob períodos mais curtos, representam formas clássicas de minimizar os problemas decorrentes da pneumonia micoplasmática. Neste sentido, uma diversidade de princípios ativos tradicionais e outros mais novos se apresentam disponíveis no mercado, sendo importante conciliar no seu uso a relação custo/ benefício.

A Tilmicosina é um dos novos antibióticos da família dos macrolídeos que apresenta elevada eficácia contra os agentes que causam a forma primária e secundária da pneumonia, sendo indicada a dose de 200 a 400 ppm. Esta droga quando absorvida apresenta de 50 a 70 vezes mais concentração intracelularmente do que extracelularmente. Os agentes patógenos quando são englobados pelos macrófagos dos pulmões recebem, portanto, uma alta concentração da Tilmicosina que destrói o agente pela inibição da síntese protéica.

Baseados na ação desta droga e nos resultados obtidos em condições a campo com doses inferiores de Tilmicosina, o objetivo deste trabalho foi avaliar a eficiência da utilização desta droga sob menor concentração no controle de doenças respiratórias nas fases de crescimento e terminação comparada com uma concentração indicada na literatura e comparada com uma combinação de dois outros antibióticos comerciais.

\section{Material e Métodos}

Os experimentos foram conduzidos em duas granjas comerciais denominadas A e B, localizadas, respectivamente, nos municípios de Ibiporã e Londrina, Estado do Paraná.

As unidades de produção de cada granja consistiam em ciclo completo com 200 e 170 matrizes híbridas comerciais e apresentavam escalas produtivas em all in all out nas fases de maternidade e creche e manejo contínuo nas demais fases.

Os experimentos em ambas as granjas foram iniciados por ocasião da transferência dos leitões das unidades de creche para a fase de crescimento (entre 60 a 70 dias de idade), sendo concluído ao final da fase de terminação (entre 130 e 135 dias de idade).

Os animais (machos castrados e fêmeas) foram mantidos em baias de crescimento e terminação para 30 animais em média, com piso compacto com lâmina d'água ao fundo, sendo preservadas densidades (área/animal) normais para as fases de crescimento e terminação.

Foram avaliados 639 animais na granja A, com peso médio inicial de 21,42 kg e idade média de 58,78 dias, e 306 animais na granja B, com peso médio 
inicial de 30,58 kg e idade média de 69,16 dias. Os animais receberam rações (a base de milho e farelo de soja) produzidas na propriedade e elaboradas com núcleos comerciais, atendendo ao mínimo as exigências nutricionais específicas para cada fase (NATIONAL RESEARCH COUNCIL, 1998).

Os tratamentos foram orientados nas fases de crescimento e terminação, correspondendo a: T1- Ração com 100 ppm de Tilmicosina; T2 - Ração com 200 ppm de Tilmicosina; e T3 - Ração com 50 ppm de Tiamulina mais 150 ppm de Cloridrato de Oxitetraciclina. Cada tratamento foi implementado no início da fase de crescimento, se estendendo a medicação por 14 dias. Os tratamentos foram retomados no início da fase de terminação por mais 14 dias (reiniciados aos 91 dias na granja A e aos 98 dias na granja B). Foram avaliados, em cada fase, o ganho diário de peso, o consumo diário de ração e a conversão alimentar.

Antecedendo o início dos experimentos, para ambas as granjas, foi acompanhada uma amostra de animais terminados no abate, sendo avaliados o grau de comprometimento dos pulmões (porcentagem de área acometida por lesões respiratórias). Para a avaliação dos pulmões foi utilizado o modelo sugerido por Müller e Abbott (1986) para a classificação das áreas lesionadas, sendo: $0 \%$ de comprometimento, 1 a $10 \%, 11$ a $20 \%, 21$ a $30 \%, 31$ a $40 \%, 41$ a $50 \%$ e maior que $50 \%$ de comprometimento.

Ao final dos experimentos, todos os animais, correspondentes a cada tratamento, foram abatidos e seus pulmões foram também avaliados de acordo com o método de Müller e Abbott (1986). Foram amostrados 305 animais da granja A e 298 animais da granja B.

Para a verificação da viabilidade econômica da utilização dos tratamentos nas rações de crescimento e terminação foi desenvolvido o custo médio em ração por quilograma de peso vivo (Yi) durante o período experimental, conforme Bellaver et al. (1985).

Na seqüência, calculou-se o Índice de Eficiência Econômica (IEE) e o Índice de Custo Médio, propostos por Barbosa et al. (1992).
Os valores (preços) dos ingredientes utilizados na elaboração dos custos foram obtidos na região de Londrina no mês de maio de 2001, sendo: farelo de soja ( $\mathrm{R} \$ 0,358 / \mathrm{kg}$ ), milho grão ( $\mathrm{R} \$ 0,133 / \mathrm{kg})$, Llisina $(\mathrm{R} \$ 4,50 / \mathrm{kg})$, núcleo suínos crescimento $(\mathrm{R} \$$ $0,95 / \mathrm{kg})$ e núcleo suínos terminação $(\mathrm{R} \$ 0,80 \mathrm{~kg})$.

$\mathrm{O}$ delineamento experimental foi inteiramente casualizado, com 3 tratamentos e 8 repetições por tratamento para a granja A e com 4 repetições para a granja B. Cada baia nas fases de crescimento e terminação representou uma repetição. Aos dados foi aplicada a análise de variância, sendo as médias comparadas pelo teste de Tukey.

Para a avaliação estatística dos dados relativos às lesões pneumônicas foi aplicado o Teste do quiquadrado.

\section{Resultados e Discussão}

Os resultados de desempenho observados nas fases de crescimento, terminação e no período total (crescimento e terminação), para as granjas A e B, estão apresentados nas Tabelas 1 e 2, respectivamente.

Tabela 1. Resultados (médias e desvio padrão) do desempenho de leitões nas fases de crescimento, terminação e no período total na granja A, submetidos aos tratamentos experimentais.

\begin{tabular}{lccc}
\hline Tratamentos & T1 & T2 & T3 \\
\hline Parâmetros & \multicolumn{3}{c}{ Fase de Crescimento } \\
\hline GPD $(\mathrm{kg})$ & $0,626 \mathrm{a} \pm 0,08$ & $0,633 \mathrm{a} \pm 0,08$ & $0,624 \mathrm{a} \pm 0,08$ \\
CDR $(\mathrm{kg})$ & $1,414 \mathrm{a} \pm 0,20$ & $1,427 \mathrm{a} \pm 0,19$ & $1,369 \mathrm{a} \pm 0,11$ \\
CA & $2,25 \mathrm{a} \pm 0,21$ & $2,25 \mathrm{a} \pm 0,04$ & $2,19 \mathrm{a} \pm 0,11$ \\
\hline \multicolumn{4}{c}{ Fase de Terminação } \\
\hline GPD (kg) & $0,920 \mathrm{a} \pm 0,12$ & $0,937 \mathrm{a} \pm 0,09$ & $0,876 \mathrm{a} \pm 0,06$ \\
CDR $(\mathrm{kg})$ & $2,371 \mathrm{a} \pm 0,36$ & $2,391 \mathrm{a} \pm 0,26$ & $2,294 \mathrm{a} \pm 0,24$ \\
CA & $2,57 \mathrm{a} \pm 0,12$ & $2,55 \mathrm{a} \pm 0,09$ & $2,62 \mathrm{a} \pm 0,25$ \\
\hline \multicolumn{4}{c}{ Período Total } \\
\hline GPD $(\mathrm{kg})$ & $0,803 \mathrm{a} \pm 0,09$ & $0,815 \mathrm{a} \pm 0,06$ & $0,773 \mathrm{a} \pm 0,05$ \\
CDR $(\mathrm{kg})$ & $1,989 \mathrm{a} \pm 0,28$ & $2,004 \mathrm{a} \pm 0,18$ & $1,914 \mathrm{a} \pm 0,20$ \\
CA & $2,47 \mathrm{a} \pm 0,10$ & $2,46 \mathrm{a} \pm 0,07$ & $2,47 \mathrm{a} \pm 0,18$ \\
\hline
\end{tabular}

*médias seguidas de mesma letra, nas linhas, não diferem entre si pelo teste de Tukey $(\mathrm{P}>0,05)$ 
Silva, C. A. da; Hoshi, E. H.; Sarubbi, J.

Tabela 2. Resultados (médias e desvio padrão) do desempenho de leitões nas fases de crescimento, terminação e no período total na granja B, submetidos aos tratamentos experimentais

\begin{tabular}{lccc}
\hline Tratamentos & T1 & T2 & T3 \\
\hline Parâmetros & \multicolumn{3}{c}{ Fase de Crescimento } \\
\cline { 2 - 4 } GPD (kg) & $0,868 \mathrm{a} \pm 0,10$ & $0,899 \mathrm{a} \pm 0,12$ & $0,856 \mathrm{a} \pm 0,11$ \\
CDR (kg) & $2,137 \mathrm{a} \pm 0,22$ & $2,151 \mathrm{a} \pm 0,07$ & $2,211 \mathrm{a} \pm 0,22$ \\
CA & $2,47 \mathrm{a} \pm 0,20$ & $2,42 \mathrm{a} \pm 0,28$ & $2,59 \mathrm{a} \pm 0,19$ \\
\hline \multirow{3}{*}{ Fase de Terminação } \\
\cline { 2 - 4 } GPD (kg) & $0,981 \mathrm{a} \pm 0,06$ & $1,002 \mathrm{a} \pm 0,05$ & $0,964 \mathrm{a} \pm 0,07$ \\
CDR (kg) & $2,873 \mathrm{a} \pm 0,22$ & $2,896 \mathrm{a} \pm 0,22$ & $2,718 \mathrm{a} \pm 0,21$ \\
CA & $2,93 \mathrm{a} \pm 0,22$ & $2,89 \mathrm{a} \pm 0,09$ & $2,83 \mathrm{a} \pm 0,28$ \\
\hline \multicolumn{4}{c}{ Período Total } \\
\hline GPD $(\mathrm{kg})$ & $0,925 \mathrm{a} \pm 0,41$ & $0,947 \mathrm{a} \pm 0,75$ & $0,910 \mathrm{a} \pm 0,74$ \\
CDR (kg) & $2,485 \mathrm{a} \pm 0,21$ & $2,518 \mathrm{a} \pm 0,12$ & $2,384 \mathrm{a} \pm 0,18$ \\
CA & $2,69 \mathrm{a} \pm 0,16$ & $2,66 \mathrm{a} \pm 0,14$ & $2,63 \mathrm{a} \pm 0,22$ \\
\hline
\end{tabular}

* médias seguidas de mesma letra, nas linhas, não diferem entre si pelo teste de Tukey $(\mathrm{P}>0,05)$

Pode-se observar que, independente dos tratamentos, tanto para granja A quanto para granja B, para os três parâmetros avaliados, ganho de peso diário, consumo médio de ração e conversão alimentar, nos diferentes períodos experimentais, não houve diferença significativa $(\mathrm{P}>0,05)$ entre os tratamentos.

Estes primeiros resultados permitem afirmar que os animais tratados com 100 ppm de tilmicosina (T1) apresentaram o mesmo desempenho comparado com os tratamentos cujas dosagens seguiam as recomendações técnicas e comercialmente indicadas (T2 e T3).

Quanto a classificação das lesões respiratórias observadas ao abate antes dos tratamentos experimentais e após a implantação dos tratamentos, os dados encontram-se listados nas Tabelas 3 e 4, para as granjas A e B, respectivamente.
Tabela 3. Valores percentuais de animais com lesões pulmonares ao abate antes dos tratamentos e após o estabelecimento dos tratamentos experimentais (Granja A)

\begin{tabular}{ccccc}
\hline TRATAMENTOS & $\begin{array}{c}\text { Controle } \\
\text { Pré- tratamento } \\
\text { (b)* }\end{array}$ & T1 (a) & T2 (a) & T3 (a) \\
\cline { 1 - 3 } Nível das lesões & & & & \\
\hline $0 \%$ & $30,55 \%$ & $42,57 \%$ & $43,43 \%$ & $44,76 \%$ \\
$1-10 \%$ & $38,88 \%$ & $52,47 \%$ & $46,46 \%$ & $48,57 \%$ \\
$11-20 \%$ & $22,22 \%$ & $4,95 \%$ & $10,10 \%$ & $6,66 \%$ \\
$21-30 \%$ & $5,55 \%$ & 0 & 0 & 0 \\
$31-40 \%$ & 0 & 0 & 0 & 0 \\
$41-50 \%$ & $2,77 \%$ & 0 & 0 & 0 \\
$>50 \%$ & 0 & 0 & 0 & 0 \\
\hline
\end{tabular}

* letras semelhantes entre os tratamentos indicam ausência de diferença $(\mathrm{P}>0,05)$ pelo teste de qui-quadrado. Letras distintas entre os grupos tratados e o grupo controle indicam diferença $(\mathrm{P}<0,05)$ pelo teste de qui-quadrado.

Tabela 4. Valores percentuais de animais com lesões pulmonares ao abate antes dos tratamentos e após o estabelecimento dos tratamentos experimentais (Granja B)

\begin{tabular}{|c|c|c|c|c|}
\hline TRATAMENTOS & $\begin{array}{c}\text { Controle } \\
\text { Pré- tratamento } \\
\text { (b)* }\end{array}$ & T1 (a) & T2 (a) & T3 (a) \\
\hline \multicolumn{5}{|l|}{ Nível das lesões } \\
\hline $0 \%$ & $88,00 \%$ & $89,10 \%$ & $90,62 \%$ & $91,10 \%$ \\
\hline $1-10 \%$ & $12,00 \%$ & $10,90 \%$ & $9,38 \%$ & $8,90 \%$ \\
\hline $11-20 \%$ & 0 & 0 & 0 & 0 \\
\hline $21-30 \%$ & 0 & 0 & 0 & 0 \\
\hline $31-40 \%$ & 0 & 0 & 0 & 0 \\
\hline $41-50 \%$ & 0 & 0 & 0 & 0 \\
\hline$>50 \%$ & 0 & 0 & 0 & 0 \\
\hline
\end{tabular}

* letras semelhantes entre os tratamentos indicam ausência de diferença $(\mathrm{P}>0,05)$ pelo teste de qui-quadrado. Letras distintas entre os grupos tratados e o grupo controle indicam diferença $(\mathrm{P}<0,10)$ pelo teste de quiquadrado

É possível observar que para a granja A (Tabela 3), a incidência de problemas respiratórios com características macroscópicas de lesões por micoplasma (áreas hepatizadas nos pulmões), portanto, caracterizando a pneumonia enzoótica, foi bem mais elevada que comparada com a granja $\mathrm{B}$ (Tabela 4), não obstante, entre tratamentos, tanto para a granja A quanto para a granja $\mathrm{B}$, a distribuição das lesões foi parecida entre os grupos T1, T2 e T3, não apresentando diferença estatística $(\mathrm{P}>0,05)$ entre si. Todavia, em ambas as granjas, quando comparados os animais sem lesão com os animais com lesão nos pulmões, ou seja, após os tratamentos, verificou-se, conforme as Tabelas 3 e 4, diferença entre os grupos antes e após os tratamentos. 
Como manejo regular, a Granja A praticava a vacinação contra pneumonia enzoótica aos 21 e aos 35 dias de idade. Entretanto, alguns requisitos básicos ligados aos conceitos de biosseguridade, como a limitação de pessoas alheias à granja não era respeitada, como também a utilização de vestimentas (roupas e calçados) da própria granja e o banho antes do acesso às instalações.

As edificações das fases de creche, crescimento e terminação na granja $\mathrm{A}$ eram antigas, apresentando características construtivas não condizentes com os padrões sanitários e com o conforto animal estabelecidos, como pé-direito baixo, e na creche, abertura das paredes laterais (portas e janelas) com área inferior a $20 \%$. Os barracões destas fases praticamente guardavam uma distância muito pequena entre si (em torno de 3,5 metros), insuficiente para permitir adequada ventilação e, portanto, a renovação de ar nos ambientes. Tais características identificam-se com as proposições citadas por Piffer et al. (1998) que trata da predisposição maior que se estabelece às pneumonias quando as condições ambientais são desfavoráveis.

$\mathrm{Na}$ granja B, os animais de reposição, sem exceção, eram provenientes de granjas com o mínimo de doenças (Minimal Diseases) ou livres de agentes patogênicos específicos (Specific Pathogenic Free). Sobestiansky et al. (1999) citam esses critérios como relevantes para a manutenção da granja livre de pneumonia. Paralelamente, alguns critérios de biosseguridade eram também extremamente rigorosos, como o isolamento da área de produção através de cercas, a exigência de trocas de roupas e calçados para adentrar às instalações e a proibição de acesso de pessoas alheias ao dia a dia da granja às instalações.

Também representava uma medida preventiva de rotina na granja B, a monitoria sorológica para enfermidades reprodutivas. Vale ressaltar que os prédios eram distintos por fases e guardavam uma boa distância entre si, permitindo adequada renovação de ar nos ambientes.
Na granja B, além de uma pequena redução no quadro de lesões pneumônicas, é importante registrar que o perfil das lesões após os tratamentos manteve a granja sem um quadro clínico aparente de doenças respiratórias, inclusive com um escore de lesões sob valores próximos do limite mínimo na escala de 1$10 \%$, conforme protocolo de avaliação de lesões pneumônicas.

Para a granja A, vale ressaltar a grande melhora observada em relação ao quadro de lesões pneumônicas verificadas antes da introdução dos tratamentos.

O baixo número de animais com lesões nos pulmões e a ausência de um histórico clínico de diarréia e sinais de tosse e espirros na granja B identificam-se com o perfil sanitário, zootécnico e ambiental superior que a propriedade $\mathrm{B}$ apresenta comparada com a granja $\mathrm{A}$.

Com relação as taxas de mortalidade para a granja A, os níveis, expressos percentualmente, foram semelhantes estatisticamente $(\mathrm{P}>0,01)$ para os tratamentos com Tilmicosina a 100 ppm (T1), a 200 ppm (T2) e para o grupo tratado com Tiamulina 50ppm mais Cloridrato de Oxitetraciclina 150ppm (T3), sendo iguais a $0,93 \%, 0,92 \%$ e $0,95 \%$, respectivamente, ou seja, as taxas estavam dentro dos limites estabelecidos por Sobestiansky et al. (1999). Para a granja B não foram registradas mortalidade nos tratamentos.

Quanto a viabilidade econômica do uso dos tratamentos estabelecidos, as rações (computadas as fases de crescimento e terminação) apresentaram custos diferenciados para as granjas A e B.

Confirmando os benefícios do tratamento com Timilcosina a 100 ppm (T1), o Índice de Eficiência Econômica e o Índice de Custos Médios demonstraram os melhores resultados para a menor concentração da droga nas rações (Tabela 5). Tais valores representaram uma vantagem econômica entre os limites de 2,73 e 7,62\%, de acordo com o tratamento empregado. 
Silva, C. A. da; Hoshi, E. H.; Sarubbi, J.

Tabela 5. Custo médio em ração por quilograma de peso vivo ganho por leitão no período total, Índice Médio de Custo e Índice de Eficiência Econômica, de acordo com os tratamentos experimentais.

\begin{tabular}{|c|c|c|c|}
\hline TRATAMENTOS & T1 & $\mathrm{T} 2$ & T3 \\
\hline Granja A & & & \\
\hline Custo em ração ( $\mathrm{R} \$ / \mathrm{kg})$ & 0,695 & 0,748 & 0,714 \\
\hline Índice Médio de Custo & 100,00 & 92,91 & 97,33 \\
\hline $\begin{array}{l}\text { Índice de Eficiência } \\
\text { Econômica }\end{array}$ & 100,00 & 107,62 & 102,73 \\
\hline Granja B & & & \\
\hline Custo em ração ( $\mathrm{R} \$ / \mathrm{kg})$ & 0,752 & 0,802 & 0,773 \\
\hline Índice Médio de Custo & 100,00 & 93,76 & 97,28 \\
\hline $\begin{array}{l}\text { Índice de Eficiência } \\
\text { Econômica }\end{array}$ & 100,00 & 106,64 & 102,79 \\
\hline
\end{tabular}

\section{Conclusão}

A Tilmicosina na concentração de 100 ppm foi tão eficiente quanto a Tiamulina na dosagem de 50 ppm mais Cloridrato de Oxitetraclina na dosagem de 150 ppm na redução de lesões pulmonares e nos resultados de desempenho obtidos nas fases de crescimento e terminação.

Os custos dos tratamentos favoreceram o uso da Tilmicosina na concentração de 100ppm.

\section{Agradecimentos}

A Elanco Saúde Animal, divisão da Eli Lilly do Brasil Ltda., especialmente ao Dr. Rogério Assis pela colaboração e constante atenção dispensadas na execução deste trabalho.

\section{Referências}

BARBOSA, H. P.; FIALHO, E. T.; FERREEIRA, A. S.; LIMA, G. J. M.; GOMES, M. F. M. Triguilho para suínos nas fases inicial de crescimento, crescimento e terminação. Revista Brasileira de Zootecnia, Viçosa, v.21, n.5, p.82737, 1992 .
BELLAVER, C.; FIALHO, E. T.; PROTAS, J. F. S.; GOMES, P. C. Radícula de malte na alimentação de suínos em crescimento e terminação. Pesquisa Agropecuária Brasileira, Brasilia, v.20, n.8, p.969-74, 1985.

MÜLLER, R.D., ABBOTT, P.H. Estimating the cost of respiratory disease in hogs. Animal Health Natur., Mount Morris, v.41, n.2, p.31-34, 1986.

NATIONAL RESEARCH COUNCIL. Nutrient requeriments of swine. $9^{\text {thed. Washington: National }}$ Academy Press, 1998.

PIFFER, I. A. Pneumonia enzoótica dos suínos. Concórdia: EMBRAPA-CNPSA, 1983. (Circular Técnica, 5).

PIFFER, I. A.; PERDOMO, C. C.; SOBESTIANSKY, J. Efeito de fatores ambientais na ocorrência de doenças. In: SOBESTIANSKY, J.; WENTZ, I.; SILVEIRA, P.R. S.; SESTI, L. A. C. Suinocultura intensiva. Concórdia: EMBRAPACNPSA, 1998. Cap.13, p.255-273.

RISTOW, L. E.; FÓSCOLO, C. B.; SILVA, A. F. Vacinação contra Mycoplasma hyopneumoniae: estudo comparativo de resposta a vacinas frente a influência da imunidade passiva. In: CONGRESSO BRSILEIRO DE VETERINÁRIOS ESPECIALISTAS EM SUÍNOS, 10., 2001, Porto Alegre. Anais... Porto Alegre: ABRAVES, 2001. v.2, p. $15-16$.

SILVA, A. F.; PAGANINI, F. J.; ACOSTA, J. C.; ROCHA, P. H.; MISTURA, H.; MARCON, E.; SIMON, V. A.; CASAGARANDE, H. A. Programa de gerenciamento de doenças respiratórias em suínos. II - Avaliação do programa econômico Coopers no controle da pneumonia enzoótica no Brasil. In: CONGRESSO BRASILEIRO DE VETERINÁRIOS ESPECIALISTAS EM SUÍNOS, 10, 2001. Porto Alegre. Anais... Porto Alegre: ABRAVES, 2001. v.2, p. 33-34.

SOBESTIANSKY, J.; BARCELLOS, D.; MORES, N.; CARVALHO, L. F.; OLIVEIRA, S.; MORENO, A. M.; ROEHE, P. M. Clínica e patologia suína. 2.ed. Goiânia: Ed. Art 3, 1999.

STEVENSON,W. Bacterial pneumonia in swine. In: INTERNATIONALPIG VETERINARY SOCIETY, 15., 1998, Birmingham. Proceedings...: Birmingham, 1988. v.1, p.11-20. 